\title{
FLORÍSTICA, VOLUME E BIOMASSA LENHOSA DE UM FRAGMENTO DE MATA ATLÂNTICA NO MUNICÍPIO DE SANTA MARIA DE JETIBÁ, ESPÍRITO SANTO
}

\author{
José Imaña-Encinas ${ }^{1}$, José Elias de Paula ${ }^{2}$, Cláudio de Almeida Conceição ${ }^{3}$ \\ ${ }^{1}$ Eng. Florestal, Ph.D., Depto. Engenharia Florestal, UnB, Brasília, DF, Brasil - imana @unb.br \\ ${ }^{2}$ Botânico, Dr., Depto. de Botânica, UnB, Brasília, DF, Brasil - depaula@unb.br \\ ${ }^{3}$ Biólogo, Depto. de Botânica, UFMS, Campo Grande, MS, Brasil \\ Recebido para publicação: 19/03/2010 - Aceito para publicação: 03/04/2012
}

\begin{abstract}
Resumo
Este trabalho teve como objetivo avaliar a florística, volume e biomassa em duas áreas de Mata Atlântica. Essas áreas estão localizadas na Reserva Biológica Sítio Palmares, no município de Santa Maria de Jetibá, Espírito Santo. Foram avaliadas 6 (seis) parcelas de áreas preservadas (mata primária) e 6 (seis) parcelas em uma área de regeneração natural com 33 anos de idade (mata secundária). Foram medidas todas as árvores com diâmetro a $1,30 \mathrm{~m}$ do solo (DAP) igual ou superior a $5 \mathrm{~cm}$. Na área de mata primária foram encontradas 341 árvores distribuídas em 70 espécies e 32 famílias, que apresentaram DAP médio aritmético de $14,82 \mathrm{~cm}$ e área basal igual a $43,60 \mathrm{~m}^{2} \cdot \mathrm{ha}^{-1}$, contabilizando $518 \mathrm{~m}^{3} \cdot \mathrm{ha}^{-1}$ de fustes e galhos e $397.674 \mathrm{~kg} . \mathrm{ha}^{-1}$ de biomassa lenhosa. A área de mata secundária apresentou 195 árvores classificadas em 17 espécies e 14 famílias, com DAP médio de 12,4 cm. Ainda, foram apresentados $12,5 \mathrm{~m}^{2} \cdot \mathrm{ha}^{-1}$ de área basal, $93 \mathrm{~m}^{3} \cdot \mathrm{ha}^{-1}$ de volume de madeira e $54.631 \mathrm{~kg}^{-h^{-1}}$ de biomassa lenhosa.

Palavras-chave: Levantamento florístico; biometria florestal.
\end{abstract}

\begin{abstract}
Wood volume and biomass of a fragment of the Mata Atlântica at Santa Maria de Jetibá county, state of Espirito Santo, Brazil. The paper analyzed the floristic, wood volume and their biomass in two areas of the Atlantic Forest, located at the Reserva Biológica Sítio Palmares in Santa Maria de Jetibá county in state of Espirito Santo. It was measure each six plots in one preserved areas (primary forest) and in one 33 years old on natural regeneration process (secondary forest). It was measured all the trees with over 5 $\mathrm{cm} \mathrm{DBH}$. At the area primary forest were found 341 trees distributed in 70 species and 32 families. The tree population presented an average of $14.82 \mathrm{~cm} \mathrm{DBH}$ and a basal area of $43.60 \mathrm{~m}^{2} \mathrm{ha}^{-1}$, corresponding to wood trunk and branches of $518 \mathrm{~m}^{3} \mathrm{ha}^{-1}$ equivalent to $397,674 \mathrm{~kg} \mathrm{ha}^{-1}$. The secondary forest on natural regeneration had 195 trees distributed in 17 species and 14 families, with a DBH of $12.4 \mathrm{~cm}$. The basal area was $12.5 \mathrm{~m}^{2} \mathrm{ha}^{-1}$, corresponding to $93 \mathrm{~m}^{3} \mathrm{ha}^{-1}$ of wood volume and $54,631 \mathrm{~kg} \mathrm{ha}^{-1}$ of biomass. Keywords: Floristic survey; forest biometrics.
\end{abstract}

\section{INTRODUÇÃO}

As várias fitofisionomias da Mata Atlântica estão relacionadas a fatores naturais e antrópicos e há pouco mais de cinco séculos se estendiam por toda a costa atlântica brasileira, sendo que atualmente estão restritas principalmente a áreas serranas costeiras das regiões Sul e Sudeste do Brasil (DELDUQUE, 2008). Segundo o SOS Mata Atlântica (2008), na região Sudeste, a Mata Attântica cobria em 2008 11,8\% de sua superfície original. Atualmente está reduzida a 111 milhões de hectares (SERVIÇO FLORESTAL BRASILEIRO (SFB), 2010).

A revisão bibliográfica constatou a existência de poucos trabalhos publicados relativos à cubagem, volumetria e determinação de biomassa arbórea, em locais da Mata Atlântica, evidenciando assim a importância de se realizarem estudos correlatos sobre a volumetria e a biomassa lenhosa dessa formação vegetal. A literatura registra os trabalhos de Souza e Jesus (1991), Chichorro et al. (2003) e Rolim et al. (2006a), que definiram equações de volume; Burger e Delitti (2008), Rolim et al. (2005), Silveira et al. (2008), Vieira et al. (2008) e Cunha et al. (2009), que realizaram estudos para a determinação de biomassa em áreas de Mata Atlântica por meio de modelos alométricos.

A literatura registra, em relação a estudos ecológicos, pedológicos, florísticos e fitossociológicos da Mata Atlântica, vários trabalhos, porém, orientados à dinâmica e estruturas de crescimento da 
correspondente regeneração ou sucessão natural de comunidades arbóreas, só foram encontrados os realizados por Drumond e Meira Neto (1999), Marangon et al. (2008) e Aparício et al. (2011).

Justifica-se, portanto, a elaboração do presente estudo, tendo em vista que a Mata Atlântica é um dos ecossistemas mais antropizados do Bioma Costeiro ou Atlântico, registrando a real situação comparativa em termos de espécies arbóreas e da produção madeireira de uma área que não sofreu intervenção humana com uma em fase de secundarização. Nesse sentido, o estudo teve por objetivo realizar o levantamento florístico arbóreo e dendrométrico de duas áreas de floresta atlântica, uma preservada e a outra antropizada, e determinar o volume de madeira e a correspondente biomassa lenhosa.

\section{MATERIAL E MÉTODOS}

A área de estudo está localizada na Reserva Biológica Sítio Palmares, Distrito Rio Claro, do Município de Santa Maria Jetibá, Estado do Espírito Santo, em uma altitude de $692 \mathrm{~m}$.

O clima da região, segundo a classificação de Köppen, é do tipo tropical de altitude Cwb, com estações chuvosas e secas bem definidas. Na estação fria, a temperatura varia entre 7 e $9{ }^{\circ} \mathrm{C}$, e nos meses mais quentes entre 25 e $27{ }^{\circ} \mathrm{C}$, com temperatura média anual de $18{ }^{\circ} \mathrm{C}$. A precipitação média anual é de $1800 \mathrm{~mm}$ (INSTITUTO CAPIXABA DE PESQUISA, ASSISTÊNCIA TÉCNICA E EXTENSÃO RURAL (INCAPER), 2011). O solo é do tipo Cambissolo Háplico (PANOSO, 1978). A vegetação predominante da região é do tipo Floresta Ombrófila Densa.

A Reserva Biológica Sítio Palmares possui áreas preservadas e antropizadas da formação florestal Mata Atlântica. Para o presente estudo, foi escolhida aleatoriamente uma área preservada, que se denominou de mata primária, e outra em processo de regeneração natural há 33 anos, que se chamou de mata secundária.

Foram instaladas, para cada tipo de vegetação amostrada, (6) seis parcelas de $400 \mathrm{~m}^{2}$ $(20 \times 20 \mathrm{~m})$, totalizando $4.800 \mathrm{~m}^{2}$ de área amostrada. Nessas parcelas foram identificados e medidos todos os indivíduos de porte arbóreo, incluindo as palmeiras, com diâmetro a 1,30 m do solo (DAP) igual ou superior a $5 \mathrm{~cm}$. A identificação das espécies foi realizada por um especialista em dendrologia, in loco, com base em caracteres dendrológicos das folhas, flores, frutos e da casca do fuste. Nos casos em que ainda houvesse dúvidas, coletou-se material fértil, cujos binômios foram identificados no Herbário da Universidade de Brasília. As correspondentes exsicatas foram incorporadas ao acervo desse herbário. A nomenclatura dos indivíduos arbóreos identificados seguiu a classificação do $\mathrm{w}^{3}$ Tropicos do Jardim Botânico da Universidade de Missouri (TROPICOS, 2010), exceto Leguminosae, que se considerou como famílias: Caesalpiniaceae, Fabaceae e Mimosaceae.

De cada indivíduo arbóreo foi medido o DAP em $\mathrm{cm}$, e quando abatidas, foram medidos o diâmetro a $0,30 \mathrm{~m}$, que foi denominado diâmetro inferior $\left(\mathrm{D}_{\text {inf }}\right)$, o diâmetro na base da bifurcação do primeiro galho grosso $\left(\mathrm{D}_{\text {sup }}\right)$ e entre esses dois diâmetros a correspondente altura do fuste $(\mathrm{H})$, em metros. Nos galhos da copa foi utilizada a mesma metodologia, medindo-se os diâmetros na base do galho $\left(\mathrm{D}_{\text {inf }}\right)$, na base da bifurcação seguinte $\left(\mathrm{D}_{\text {sup }}\right)$ e o correspondente comprimento $(\mathrm{H})$, somente em galhos acima de $5 \mathrm{~cm}$ de diâmetro (Figura 1).

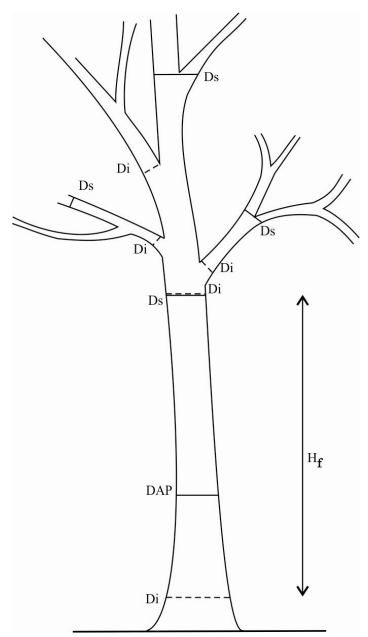

Figura 1. Pontos de medição dos diâmetros no fuste e nos galhos até um $D_{\min }=5 \mathrm{~cm}$.

Figure 1. Diameter measure points in the stem and branches, until an $D_{\min }=5 \mathrm{~cm}$. 
Para a medida dos diâmetros, foi utilizada uma suta metálica, e para o comprimento do fuste e dos galhos, uma trena de $20 \mathrm{~m}$. Para se obter o correspondente volume de madeira do fuste e dos galhos, os diâmetros medidos (em metros) foram transformados em áreas seccionais $\left(\mathrm{A}_{\mathrm{i}}\right)$, conforme expressão matemática 1 (IMAÑA-ENCINAS et al., 2002), e, junto com a altura do fuste ou comprimento do galho em metros, foram calculadas por meio da fórmula de Smalian (2).

$$
A_{i}=D_{i}^{2} \cdot 0,7854
$$

Em que: $A i=$ área seccional em $\mathrm{m}^{2}$;

$D i=$ diâmetro inferior ou superior da secção considerada;

$0,7854=\pi / 4$

$$
V=\frac{A_{1}+A_{2}}{2} \cdot H
$$

Em que: $A_{1}=$ área seccional do diâmetro inferior;

$A_{2}=$ área seccional do diâmetro superior;

$H=$ altura do fuste ou comprimento no galho.

$A_{1}$ correspondeu ao valor encontrado da área seccional do $D_{\text {inf }}$ e $A_{2}$ do $D_{\text {sup }}$, conforme mostrado na figura 1. Cada secção do galho contemplado teve seu volume calculado pela fórmula de Smalian, e a soma dos correspondentes volumes das secções obtidas forneceu o volume total dos galhos por árvore, que posteriormente foram classificados por espécie. Também foi calculada, para cada espécie encontrada, a biomassa lenhosa seca. Esse parâmetro foi obtido pela multiplicação do volume de madeira pelo correspondente valor da densidade básica de madeira. O cálculo foi realizado por árvore e sua correspondente somatória por espécie extrapolada para o hectare, como é apresentado na equação (3).

$$
B M_{L}=V\left(m^{3} / h a\right) \cdot D B\left(k g / m^{3}\right)
$$

Em que: $B M_{L}=$ biomassa lenhosa $\left(\mathrm{kg} \cdot \mathrm{ha}^{-1}\right)$;

$V=$ volume de madeira $\left(\mathrm{m}^{3} \cdot \mathrm{ha}^{-1}\right)$;

$D B=$ densidade básica da madeira $\left(\mathrm{kg} / \mathrm{m}^{3}\right)$.

Para a determinação da densidade básica da madeira das espécies encontradas no levantamento, foram extraídas amostras de madeira na altura do DAP, para representar a densidade do fuste, e na base da primeira bifurcação, para representar a densidade dos galhos. A densidade básica foi determinada pela razão do peso da amostra seca, obtida em estufa a $105^{\circ} \mathrm{C}$ (recomendado pela ABNT) sobre o volume da amostra saturada (peso constante).

A riqueza florística foi analisada pelo índice de similaridade de Jaccard (KENT; COKER, 1994), por meio da expressão 4 .

$$
S_{J}=\frac{a}{(a+b+c)}
$$

Em que: $S_{J}=$ coeficiente de similaridade de Jaccard;

$a=$ número de espécies comuns em ambas as áreas;

$b=$ número de espécies únicas na área da mata primária;

$c=$ número de espécies únicas na área da mata secundária.

A diversidade florística foi identificada pelo índice não paramétrico de Shannon-Weaver (H'), baseado na abundância proporcional das espécies (MAGURRAN, 1988), por meio das expressões 5 e 6.

$$
p_{i}=\frac{n_{i}}{N}
$$

Em que: $p_{i}=$ estimativa da proporção de indivíduos (i) encontrados de cada espécie;

$n_{i}=$ número de indivíduos da espécie "i";

$N=$ número total de indivíduos da amostra (mata primária, mata secundária). 


$$
H^{\prime}=\left(-\sum p_{i} \cdot \ln p_{i}\right)
$$

Em que: $H^{\prime}$ = índice de diversidade de Shannon-Weaver;

$\ln =$ logaritmo na base $\mathrm{n}$;

$\Sigma=$ soma de todos os "i" espécies da amostra.

\section{RESULTADOS E DISCUSSÃO}

\section{Florística}

Na tabela 1 é apresentada a densidade populacional das 77 espécies encontradas nas duas áreas de observação, mata primária e mata secundária da Reserva Biológica Sítio Palmares.

Tabela 1. Espécies e número de indivíduos arbóreos encontrados na formação de mata primária e de mata secundária em regeneração natural da Reserva Biológica Sítio Palmares.

Table 1. Number of trees of the primary forest and the secondary forest on natural regeneration at the Reserva Biológica Sítio Palmares.

\begin{tabular}{|c|c|c|c|c|}
\hline & Família / espécie & Nome popular & $\begin{array}{c}\text { Mata } \\
\text { primária }\end{array}$ & $\begin{array}{c}\text { Mata } \\
\text { secundária }\end{array}$ \\
\hline & Anacardiaceae & & & \\
\hline 01 & $\begin{array}{l}\text { Tapirira guianensis Aubl. } \\
\text { Annonaceae }\end{array}$ & pau-pombo & 8 & \\
\hline 02 & $\begin{array}{l}\text { Xylopia sericea A. St.-Hil. } \\
\text { Apocynaceae }\end{array}$ & envira & 13 & 3 \\
\hline 03 & Aspidosperma australe Mull. Arg. & peroba & 7 & \\
\hline 04 & Aspidosperma cylindrocarpon Mull. Arg. & peroba & 1 & \\
\hline 05 & Aspidosperma polyneurum Mull. Arg. & peroba & 5 & \\
\hline 06 & Aspidosperma populifolium A. DC. & peroba & 1 & \\
\hline 07 & $\begin{array}{l}\text { Aspidosperma subincanum Mart. ex A. DC. } \\
\text { Arecaceae (Palmae) }\end{array}$ & peroba & 2 & \\
\hline 08 & Euterpe edulis Mart. & palmito & 62 & 1 \\
\hline 09 & $\begin{array}{l}\text { Syagrus botryophora (Mart.) Mart. } \\
\text { Asteraceae }\end{array}$ & coco-do-mato & 3 & \\
\hline 10 & Piptocarpha macropoda (DC.) Baker & & & 18 \\
\hline 11 & $\begin{array}{l}\text { Vanillosmopsis erythropappa (DC.) Sch. Bip } \\
\text { Bignoniaceae }\end{array}$ & candeia & & 3 \\
\hline 12 & $\begin{array}{l}\text { Tabebuia cassinoides (Lam.) DC. } \\
\text { Boraginaceae }\end{array}$ & caixeta & 1 & \\
\hline 13 & Cordia sellowiana Cham. & freijó & 3 & \\
\hline 14 & $\begin{array}{l}\text { Cordia trichotoma (Vell.) Arráb. ex Steud. } \\
\text { Burseraceae }\end{array}$ & freijó & 2 & \\
\hline 15 & $\begin{array}{l}\text { Protium brasiliense Engl. } \\
\text { Caesalpiniaceae }\end{array}$ & breu & 1 & \\
\hline 16 & Apuleia leiocarpa (Vogel) J.F. Macbr. & garapa & 2 & \\
\hline 17 & Cassia ferruginea (Schrader) Schrader ex DC. & chorão & 1 & \\
\hline 18 & Cenostigma angustifolum Tul. & canela-de-velho & 3 & \\
\hline 19 & Hymenaea courbaril $\mathrm{L}$. & jatobá & 3 & \\
\hline 20 & $\begin{array}{l}\text { Sclerolobium densiflorum Benth. } \\
\text { Calophyllaceae }\end{array}$ & ingá-porco & 1 & \\
\hline 21 & $\begin{array}{l}\text { Caraipa densifolia Mart. } \\
\text { Chrysobalanaceae }\end{array}$ & camaçari & 1 & \\
\hline 22 & $\begin{array}{l}\text { Couepia rufa Ducke } \\
\text { Clusiaceae (Guttiferae) }\end{array}$ & oiti-coró & 1 & \\
\hline 23 & Rheedia brasiliensis (Mart.) Planch. \& Triana & bacupari & 1 & \\
\hline 24 & $\begin{array}{l}\text { Symphonia globulifera L. f. } \\
\text { Euphorbiaceae }\end{array}$ & anani & 1 & \\
\hline 25 & Alchornea triplinervia (Spreng) Mull. Arg. & ará-de-espinho & 1 & \\
\hline 26 & Maprounea guianensis Aubl. & cascudo & 28 & 8 \\
\hline
\end{tabular}




\begin{tabular}{|c|c|c|c|c|}
\hline & Fabaceae & & & \\
\hline 27 & Andira fraxinifolia Benth. & angelim & 2 & \\
\hline 28 & Dalbergia decipularis Rizzini \& A. Mattos & sebastião-de-arruda & 1 & \\
\hline 29 & Dalbergia nigra (Vell.) Allemao ex Benth. & jacarandá-da-bahia & 4 & 2 \\
\hline 30 & Ormosia arborea (Vell.) Harms & tento & 1 & \\
\hline 31 & Ormosia fastigiata Tul. & tento & 2 & \\
\hline 32 & Platycyamus regnelli Benth, & pau-pereira & 1 & \\
\hline 33 & Sweetia fruticosa Spreng. & sucupirana & 2 & \\
\hline 34 & $\begin{array}{l}\text { Vataireopsis araroba (Aguiar) Ducke } \\
\text { Hypericaceae }\end{array}$ & angelim-amargoso & 1 & \\
\hline 35 & $\begin{array}{l}\text { Vismia guianensis (Aubl.) Seem. } \\
\text { Hippocrateaceae }\end{array}$ & pau-lacre & & 23 \\
\hline 36 & $\begin{array}{l}\text { Salacia amygdalina Peyr. } \\
\text { Lacistemataceae }\end{array}$ & bacupari-da-mata & 20 & \\
\hline 37 & $\begin{array}{l}\text { Lacistema pubescens Mart. } \\
\text { Lauraceae }\end{array}$ & & 3 & \\
\hline 38 & Mezilaurus navalium (Allemão) Taub. Ex Mez & itaúbi & 3 & \\
\hline 39 & Nectandra myriantha Meisn. & louro & 3 & \\
\hline 40 & Nectandra reticulata (Ruiz \& Pav.) Mez & canela & 2 & \\
\hline 41 & Nectandra rigida (Kunth) Nees & louro & 1 & \\
\hline 42 & Ocotea catarinensis Mez. & canela-preta & 13 & \\
\hline 43 & Ocotea elegans Mez. & canela & 17 & 2 \\
\hline 44 & Ocotea glomerata (Nees) Mez. & louro & 8 & \\
\hline 45 & Ocotea pretiosa (Nees) Mez. & sassafrás & 15 & \\
\hline 46 & $\begin{array}{l}\text { Ocotea pulchella Mart. } \\
\text { Lecythidaceae }\end{array}$ & canela & 6 & \\
\hline 47 & $\begin{array}{l}\text { Cariniana estrellensis (Raddi) Kuntze } \\
\text { Melastomataceae }\end{array}$ & jequitibá & 3 & \\
\hline 48 & Huberia glazioviana Cong. & & 1 & \\
\hline 49 & Miconia cinnamomifolia (DC) Naudin & jacatirão-de-copada & 4 & 58 \\
\hline 50 & Miconia minutiflora (Bonpl.) DC. & quaresmeira & 7 & 1 \\
\hline 51 & $\begin{array}{l}\text { Miconia multiflora Cong. } \\
\text { Meliaceae }\end{array}$ & quaresmeira & & 25 \\
\hline 52 & Cabralea canjerana (Vell.) Mart. & canjerana & 1 & \\
\hline 53 & $\begin{array}{l}\text { Guarea guidonia }(\text { L.) Sleumer } \\
\text { Mimosaceae }\end{array}$ & jitó & 5 & 5 \\
\hline 54 & Anadenanthera macrocarpa (Benth.) Brenan & angico-vermelho & 1 & \\
\hline 55 & Enterolobium contortisiliquum (Vell.) Morong & tamboril & 1 & \\
\hline 56 & Parkia pendula (Willd.) Benth. ex Walp. & murta & 1 & \\
\hline 57 & $\begin{array}{l}\text { Piptadenia communis Benth. } \\
\text { Monimiaceae }\end{array}$ & jacaré & 1 & \\
\hline 58 & $\begin{array}{l}\text { Mollinedia gilgiana Perkins } \\
\text { Moraceae }\end{array}$ & cidreira-da-mata & 1 & \\
\hline 59 & Fícus enormis (Mart. ex Miq.) Mart. & mata-pau & 1 & \\
\hline 60 & $\begin{array}{l}\text { Sorocea bonplandii (Baill.) Burger, Lanj. \& Wess. Boer. } \\
\text { Myrtaceae }\end{array}$ & candeia & & 1 \\
\hline 61 & Eugenia involucrata DC. & & 9 & \\
\hline 62 & $\begin{array}{l}\text { Myrcia pubipetala } \text { Miq. } \\
\text { Opiliaceae }\end{array}$ & murta & 7 & \\
\hline 63 & $\begin{array}{l}\text { Agonandra brasiliensis Miers. ex Benth. \& Hook. F. } \\
\text { Phytolaccaceae }\end{array}$ & pau-marfim & 1 & \\
\hline 64 & $\begin{array}{l}\text { Gallesia gorazema (Vel.) Moq. } \\
\text { Rubiaceae }\end{array}$ & pau-d'alho & 1 & \\
\hline 65 & Coussarea friburgensis $\mathrm{M}$. Gomes & bugre-branco & 3 & \\
\hline 66 & Guettarda viburnoides Cham. \& Schltdl. & & & 1 \\
\hline 67 & $\begin{array}{l}\text { Psychotria vellosiana Benth. } \\
\text { Sapindaceae }\end{array}$ & biribi & 2 & \\
\hline 68 & $\begin{array}{l}\text { Cupania vernalis Cambess. } \\
\text { Sapotaceae }\end{array}$ & caboatá & 2 & \\
\hline
\end{tabular}




\begin{tabular}{|c|c|c|c|c|}
\hline 69 & Manilkara elata (Allemão ex Miq.) Monach. & paraju & 3 & \multirow{5}{*}{1} \\
\hline 70 & Manilkara longifolia (A. DC.) Dubard & paraju & 8 & \\
\hline 71 & $\begin{array}{l}\text { Manilkara rufula (Miq.) H.J. Lam } \\
\text { Solanacea }\end{array}$ & maçaranduba & 4 & \\
\hline 72 & $\begin{array}{l}\text { Solanum verbascifolium } \mathrm{L} \text {. } \\
\text { Sterculiaceae }\end{array}$ & trombeta & & \\
\hline 73 & $\begin{array}{l}\text { Basiloxilon brasiliensis (Allemão) K. Schum } \\
\text { Tiliaceae }\end{array}$ & pau-rei & 12 & \\
\hline 74 & $\begin{array}{l}\text { Apeiba tibourbon Aubl. } \\
\text { Urticaceae }\end{array}$ & pau-de-jangada & 1 & \multirow{3}{*}{40} \\
\hline 75 & $\begin{array}{l}\text { Cecropia catarinensis Cautrec. } \\
\text { Verbenaceae }\end{array}$ & imabauba & 1 & \\
\hline 76 & $\begin{array}{l}\text { Aegiphila sellowiana Cham, } \\
\text { Vochysiaceae }\end{array}$ & briaúba & 2 & \\
\hline 77 & Vochysia bifalcata Warm. & gomeira & 1 & 3 \\
\hline & Total & & 341 & 195 \\
\hline
\end{tabular}

O levantamento florístico das duas áreas do fragmento da Mata Atlântica estudada registrou a ocorrência de 36 famílias, 60 gêneros distribuídos em 77 espécies. A família com maior riqueza em espécies foi a Lauraceae (nove espécies), seguida pelas famílias Fabaceae e Apocynaceae, com oito e cinco espécies, respectivamente, perfazendo as três famílias $40 \%$ do total das espécies.

Na mata primária, foram encontrados 341 indivíduos provenientes de 70 espécies, distribuídas em 32 famílias. As espécies que tiveram maior presença em número de indivíduos foram Euterpe edulis (62), Maprounea guianensis (28) e Salacia amygdalina (20), perfazendo 32,26\% da comunidade observada. O número de indivíduos encontrados na mata primária foi de 1.420 árvores por hectare, valor que pode ser considerado aceitável quando comparado com o da literatura. Alves Júnior et al. (2007) registraram, para fragmentos de Mata Atlântica em Recife, de 549 a 1.657 indivíduos com DAP acima de $5 \mathrm{~cm}$ por hectare, indivíduos pertencentes a 54 espécies distribuídas em 25 famílias. Oliveira (2002) encontrou uma população de 2.784 n.ha ${ }^{-1}$ na idade de 25 anos e de 2.273 n.ha ${ }^{-1}$ na idade de 50 anos em um fragmento de maciço litorâneo da Mata Atlântica, localizado na Ilha Grande (Rio de Janeiro). Kurtz e Araújo (2000) informaram a existência de 1.370 n.ha ${ }^{-1}$ na mata da Estação Ecológica Estadual do Paraíso (RJ). Observando todas essas densidades populacionais e a correspondente riqueza florística, em função dos resultados encontrados por Kurtz e Araújo (2000) e Oliveira (2002), pode-se inferir que a estrutura da mata primária estudada provavelmente se encontra próxima da fase do clímax de desenvolvimento. Drumond e Meira Neto (1999), em um trecho da Mata Atlântica na região do Médio Rio Doce (MG), encontraram uma composição florística arbórea formada por 1.247 n.ha-1 ${ }^{-1}$ distribuídos em 43 espécies. Alves Júnior et al. (2007) encontraram, em um fragmento de Mata Atlântica em Recife, 1.098 n.ha ${ }^{-1}$, pertencentes a 54 espécies distribuídas em 25 famílias. Rolim et al. (2006b), em uma área de 20.000 ha na região de Linhares (ES), registraram 408 espécies arbóreas distribuídas em 59 famílias.

Em relação à área da mata secundária que se encontra em processo de regeneração natural, foram medidos indivíduos arbóreos de 17 espécies, distribuídas em 14 famílias. Dessas espécies, somente 15,7\% foram registradas em ambas as áreas de estudo (mata primária e secundária). Nesta área, as maiores densidades populacionais foram das espécies Miconia cinnamomifolia (58 indivíduos) e Cecropia catarinensis (40 indivíduos). As espécies Miconia multiflora e Vismia guianensis contabilizaram 25 e 23 indivíduos, respectivamente. Essas quatro espécies totalizaram $73,73 \%$ dos indivíduos arbóreos. Foram encontrados 812 indivíduos arbóreos com DAP acima de $5 \mathrm{~cm}$. Comparando com a mata primária, inferese que a mata secundária em processo de regeneração natural, após 33 anos, ainda encontra-se em uma fase inicial de estabelecimento do clímax. As quatro espécies citadas anteriormente podem ser classificadas como pioneiras da sucessão natural dessa formação vegetal.

Silva et al. (2009), no levantamento da regeneração natural em um fragmento de floresta atlântica localizada em Recife (PE), encontraram, em $2.500 \mathrm{~m}^{2}, 317$ indivíduos arbóreos com DAP acima de $5 \mathrm{~cm}$, distribuídos em 25 famílias e 48 espécies. No município de Catende (PE), em levantamento realizado sobre a estrutura da regeneração natural de um fragmento de Mata Atlântica, Aparicio et al. (2011) encontraram, em $325 \mathrm{~m}^{2}$ de área amostral, 43 espécies distribuídas em 21 famílias. No estudo do bosque secundário de um fragmento de floresta estacional semidecidual em Viçosa (MG), Marangon et al. (2008) encontraram, em $1.000 \mathrm{~m}^{2}, 957$ indivíduos arbóreos classificados em 91 espécies pertencentes a 30 famílias. As densidades populacionais informadas contribuem para a confirmação de que a área da 
mata secundária, em regeneração natural, da Reserva Biológica Sítio Palmares ainda está em uma fase inicial da sucessão natural para atingir o clímax correspondente.

Analisando a riqueza florística pelo índice ou coeficiente de similaridade de Jaccard (MUELLER-DOMBOIS; ELLENBERG, 1974), obteve-se um valor de 0,5, que identifica que ambas as comunidades, mata primária e secundária, são bastante diferentes em relação às espécies, que apresentou 70 e 17 espécies para a mata primária e secundária, respectivamente, e 0,44 entre famílias. O índice de Shannon-Weaver forneceu uma baixa diversidade de espécies, correspondendo ao valor de 2,68 nats.ind ${ }^{-1}$, corroborando os resultados apresentados, que ficaram dentro dos padrões (H' entre 1,69 e 4,4) de crescimento conhecidos para a Mata Atlântica (KURTZ; ARAÚJO, 2000). Borém e Oliveira Filho (2002) encontraram índices pertinentes entre 3,6 e 4,1 para uma topossequência de uma mata secundária com 30 anos de idade localizada no município de Silva Jardim, no estado do Rio de Janeiro.

\section{Diâmetro e área basal}

Ao avaliar o diâmetro das árvores, foi encontrado o DAP médio de $14,82 \mathrm{~cm}$ para a mata primária e $12,40 \mathrm{~cm}$ para a mata secundária. Pode-se inferir que ambas as comunidades vegetais possuem estrutura arbórea com indivíduos de porte pequeno e médio. Pela classificação silvicultural que considera o tamanho do indivíduo arbóreo, segundo Beek e Sáenz (1992) e Hawley e Smith (1982), um DAP situado entre 10 e $30 \mathrm{~cm}$ está encaixado na classe fustal em florestas tropicais e sistemas de manejo de florestas secundárias tropicais. O DAP médio encontrado corresponderia à fase dos latizais, que estão no intervalo de 5 a $20 \mathrm{~cm}$, conforme o Centro de Información de Experiencias de Adaptación y Crecimiento de Especies Forestales en Chile (CIAF) (2012) e Cruz Johnson et al. (2005). Consequentemente, 43\% da comunidade vegetal observada encontra-se em fase inicial de formação dos fustais, para posteriormente entrarem esses indivíduos na fase final de árvores aproveitáveis comercialmente. Comparativamente, Borém e Oliveira-Filho (2002) registraram DAP médio de $11,4 \mathrm{~cm}$ em remanescentes alterados da Mata Atlântica no município de Silva Jardim (RJ), e Burger e Delitti (2008), na floresta secundária de 30 anos de idade do parque estadual Serra do Mar (SP), 8,5 cm.

Em relação à área basal, foram medidos $43,60 \mathrm{~m}^{2} \cdot \mathrm{ha}^{-1}$ para a mata primária e $12,52 \mathrm{~m}^{2} \cdot \mathrm{ha}^{-1}$ para a mata secundária em regeneração natural. Braga et al. (2009) encontraram, num fragmento de Mata Atlântica localizado na Reserva Florestal e Ecológica Mata do Paraíso, em Minas Gerais, áreas com área basal variando de $21,17 \mathrm{~m}^{2} \cdot \mathrm{ha}^{-1}$ a $60,35 \mathrm{~m}^{2} \cdot \mathrm{ha}^{-1}$. Kurtz e Araújo (2000) registraram, para um trecho de Mata Atlântica no estado de Rio do Janeiro, 57,28 $\mathrm{m}^{2}$.ha ${ }^{-1}$. Oliveira (2002) registrou área basal de $26,3 \mathrm{~m}^{2} \cdot \mathrm{ha}^{-1}$ e $32,4 \mathrm{~m}^{2} \cdot \mathrm{ha}^{-1}$ em áreas de 25 e 50 anos de idade, respectivamente, em um fragmento litorâneo de Mata Atlântica em Ilha Grande, Rio de Janeiro. Drumond e Meira Neto (1999) registraram, em uma área após 25 anos de regeneração natural, na região do médio Rio Doce, Minas Gerais, uma área basal igual a $16,7 \mathrm{~m}^{2} \cdot \mathrm{ha}^{-1}$. Borém e Oliveira-Filho (2002) encontraram, em uma topossequência alterada de Mata Atlântica no município de Silva Jardim, no Rio de Janeiro, entre 23,15 e 33,34 m².ha ${ }^{-1}$. Na mata primária, a distribuição segue o padrão típico de "J invertido" para as comunidades vegetais naturais. Na aérea em regeneração natural, mata secundária, a densidade populacional mostrou um comportamento atípico de distribuição de seus indivíduos arbóreos, como é mostrado na figura 2.
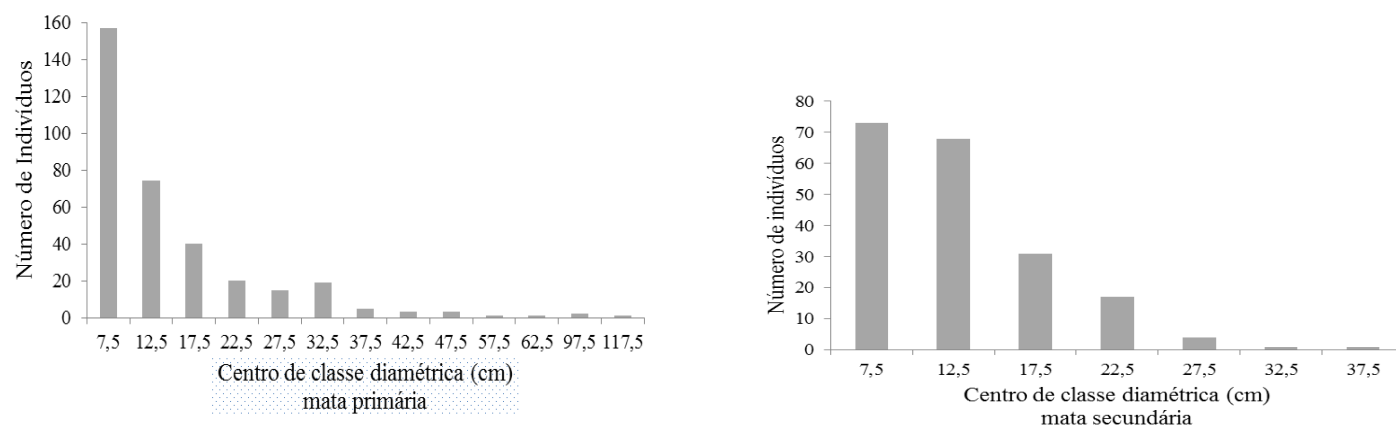

Figura 2. Distribuição diamétrica das comunidades observadas na Reserva Biológica Sítio Palmares.

Figure 2. Diametric distribution of the observed communities at Reserva Biológica Sítio Palmares.

\section{Volume e biomassa}

Na tabela 2 são apresentados os parâmetros relativos ao cálculo de volume de madeira e biomassa lenhosa, especificados pelas 73 espécies encontradas nas duas áreas de estudo. 
Na mata primária foi contabilizado um volume total de madeira igual a $124,60 \mathrm{~m}^{3}$ (Tabela 2), sendo $84,34 \%$ de volume dos fustes e $15,65 \%$ dos galhos, correspondendo a $518,34 \mathrm{~m}^{3} \cdot \mathrm{ha}^{-1}$.

As espécies que apresentaram maior volume de madeira, na mata primária, foram Manilkara longifolia $\left(11,27 \mathrm{~m}^{3}\right)$, Aspidosperma australe $\left(8,36 \mathrm{~m}^{3}\right)$, Maprounea guianensis $\left(7,82 \mathrm{~m}^{3}\right)$, Tapira guianensis $\left(7,63 \mathrm{~m}^{3}\right)$ e Basiloxilon brasiliensis $\left(7,00 \mathrm{~m}^{3}\right)$. Essas cinco espécies, com 63 indivíduos arbóreos (18\% das árvores), acumularam $42,08 \mathrm{~m}^{3}$ (33,77\% do volume total). Além disso, foi observado que 37 espécies (53\%) apresentaram volume de madeira inferior a $1 \mathrm{~m}^{3} .13$ espécies $(18,6 \%)$ com pequeno volume de copa, cujos ramos possuíam diâmetros menores a $5 \mathrm{~cm}$, não apresentaram correspondente volume de madeira dos galhos (Tabela 2).

Tabela 2. Parâmetros dendrométricos das espécies arbóreas encontradas na formação da mata primária na Reserva Biológica Sítio Palmares. Numeração da espécie extraída da tabela 1.

Table 2. Dendrometrical parameters of the woody species meet on the primary forest formation at Reserva Biológica Sitio Palmares. Species number took from table 1.

\begin{tabular}{|c|c|c|c|c|c|c|c|c|c|c|c|}
\hline \multirow[b]{2}{*}{ Espécie } & \multirow{2}{*}{$\begin{array}{c}\text { Densidade } \\
\text { Básica } \\
\left.(\mathbf{k g . m})^{3}\right)\end{array}$} & \multirow[b]{2}{*}{$\mathbf{n}$} & \multirow{2}{*}{$\begin{array}{l}\text { DAP } \\
\text { médio } \\
(\mathrm{cm})\end{array}$} & \multicolumn{4}{|c|}{ Volume } & \multicolumn{4}{|c|}{ Biomassa } \\
\hline & & & & $\begin{array}{c}\text { Fuste } \\
\left(\mathbf{m}^{3}\right)\end{array}$ & $\begin{array}{c}\text { Galhos } \\
\left(\mathbf{m}^{3}\right)\end{array}$ & $\begin{array}{c}\text { Total } \\
\left(\mathbf{m}^{3}\right)\end{array}$ & $\begin{array}{l}\text { Madeira } \\
\left(\mathrm{m}^{3} \mathrm{ha}^{-1}\right)\end{array}$ & $\begin{array}{c}\text { Fuste } \\
\text { (kg) }\end{array}$ & $\begin{array}{c}\text { Galhos } \\
\text { (kg) }\end{array}$ & $\begin{array}{c}\text { Total } \\
\text { (kg) }\end{array}$ & $\begin{array}{c}\text { Seca } \\
\left({\left.\mathrm{kg} . h a^{-1}\right)}^{-1}\right.\end{array}$ \\
\hline 01 & 650 & 8 & 24,81 & 6,1393 & 1,4883 & 7,6276 & 31,7308 & $3.990,55$ & 418,62 & $4.957,95$ & 20625,07 \\
\hline 02 & 800 & 13 & 8,81 & 0,6989 & 0,0879 & 0,7868 & 3,2732 & 559,13 & 70,33 & 629,46 & 2618,55 \\
\hline 03 & 920 & 7 & 24,43 & 6,7267 & 1,6319 & 8,3587 & 34,7722 & $6.188,58$ & $1.501,43$ & $7.690,01$ & 31990,44 \\
\hline 04 & 660 & 1 & 30,0 & 1,1781 & 0,1791 & 1,3572 & 5,6462 & 777,55 & 118,25 & 895,8 & 3726,53 \\
\hline 05 & 898 & 5 & 11,2 & 0,6630 & 0,0208 & 0,6839 & 2,8451 & 595,41 & 18,76 & 614,17 & 2554,95 \\
\hline 06 & 894 & 1 & 17,0 & 0,3204 & 0,0430 & 0,3635 & 1,5122 & 286,48 & 38,51 & 324,99 & 1351,96 \\
\hline 07 & 880 & 2 & 9,5 & 0,0651 & & 0,0651 & 0,2711 & 57,37 & & 57,37 & 238,66 \\
\hline 08 & 798 & 62 & 8,52 & 1,8167 & & 1,8167 & 7,5575 & $1.449,73$ & & $1.449,73$ & 6030,88 \\
\hline 09 & 883 & 3 & 13,67 & 0,3595 & & 0,3595 & 1,4957 & 317,49 & & 317,49 & 1320,76 \\
\hline 12 & 502 & 1 & 21,0 & 0,8131 & 0,0621 & 0,8752 & 3,6410 & 408,19 & 31,18 & 439,37 & 1827,78 \\
\hline 13 & 793 & 3 & 19,5 & 0,9940 & 0,1779 & 1,1719 & 4,8754 & 788,31 & 141,07 & 929,38 & 3866,22 \\
\hline 14 & 684 & 2 & 22,25 & 0,9681 & 0,1652 & 1,1334 & 4,7150 & 662,22 & 113,05 & 775,27 & 3225,12 \\
\hline 15 & 600 & 1 & 31,5 & 0,8906 & 0,1281 & 1,0188 & 4,2382 & 534,39 & 76,9 & 611,29 & 2542,97 \\
\hline 16 & 989 & 2 & 23,25 & 1,5197 & 0,2587 & 1,7784 & 7,3984 & $1.503,03$ & 255,87 & $1.758,90$ & 7317,02 \\
\hline 17 & 806 & 1 & 13,0 & 0,0980 & 0,027 & 0,1256 & 0,5228 & 79 & 22,31 & 101,31 & 421,45 \\
\hline 18 & 910 & 3 & 18,67 & 0,9731 & 0,0991 & 1,0722 & 4,4607 & 885,53 & 90,25 & 975,78 & 4059,24 \\
\hline 19 & 850 & 3 & 27,67 & 3,1685 & 0,3819 & 3,5504 & 14,7700 & $2.693,22$ & 324,7 & $3.017,92$ & 12554,55 \\
\hline 20 & 804 & 1 & 13,0 & 0,1010 & & 0,1010 & 0,4204 & 81,27 & & 81,27 & 338,08 \\
\hline 21 & 820 & 1 & 20,5 & 0,4830 & 0,0480 & 0,5310 & 2,2091 & 396,08 & 39,38 & 435,46 & 1811,51 \\
\hline 22 & 982 & 1 & 66,0 & 3,2345 & 0,9046 & 4,1392 & 17,2190 & $3.176,37$ & 888,4 & $4.064,77$ & 16909,44 \\
\hline 23 & 803 & 1 & 7,0 & 0,0164 & & 0,0164 & 0,0686 & 13,24 & & 13,24 & 55,08 \\
\hline 24 & 620 & 1 & 26,5 & 0,7492 & 0,1002 & 0,8495 & 3,5340 & 464,55 & 62,16 & 526,71 & 2191,11 \\
\hline 25 & 503 & 1 & 7,5 & 0,0353 & & 0,0353 & 0,1470 & 17,78 & & 17,78 & 73,96 \\
\hline 26 & 720 & 28 & 12,52 & 6,9892 & 0,8306 & 7,8199 & 32,5307 & $5.032,29$ & 598,04 & $5.630,33$ & 23422,17 \\
\hline 27 & 782 & 2 & 15,25 & 0,9886 & 0,1207 & 1,1093 & 4,6149 & 773,13 & 94,39 & 867,52 & 3608,88 \\
\hline 28 & 899 & 1 & 47,0 & 2,3255 & 0,2561 & 2,5817 & 10,7399 & $2.090,69$ & 230,28 & $2.320,97$ & 9655,24 \\
\hline 29 & 920 & 4 & 12,87 & 0,6783 & 0,1179 & 0,7963 & 3,3129 & 624,12 & 108,55 & 732,67 & 3047,91 \\
\hline 30 & 812 & 1 & 8,0 & 0,018 & & 0,018 & 0,0784 & 15,31 & & 15,31 & 63,69 \\
\hline 31 & 790 & 2 & 18,25 & 0,7245 & 0,1286 & 0,8531 & 3,5492 & 572,38 & 101,64 & 674,02 & 2803,92 \\
\hline 32 & 910 & 1 & 27,0 & 0,8482 & 0,1283 & 0,9765 & 4,0625 & 771,89 & 116,79 & 888,68 & 3696,91 \\
\hline 33 & 993 & 2 & 37,0 & 2,3483 & 0,2073 & 2,5557 & 10,6318 & $2.331,91$ & 205,92 & $2.537,83$ & 10557,37 \\
\hline 34 & 600 & 1 & 16,0 & 0,310 & 0,0614 & 0,3717 & 1,5466 & 186,23 & 36,85 & 223,08 & 928,01 \\
\hline 36 & 600 & 20 & 9,27 & 1,4165 & 0,1861 & 1,6027 & 6,6672 & 849,95 & 111,68 & 961,63 & 4000,38 \\
\hline 37 & 709 & 3 & 8,0 & 0,1429 & 0,0107 & 0,1536 & 0,6393 & 101,35 & 7,62 & 108,97 & 453,32 \\
\hline 38 & 843 & 3 & 12,83 & 0,5811 & 0,0138 & 0,5950 & 2,4753 & 489,95 & 11,67 & 501,62 & 2086,74 \\
\hline 39 & 782 & 3 & 9,67 & 0,1909 & & 0,1909 & 0,7942 & 149,31 & & 149,31 & 621,13 \\
\hline 40 & 792 & 2 & 9,5 & 0,0702 & & 0,0702 & 0,2924 & 55,67 & & 55,67 & 231,59 \\
\hline 41 & 797 & 1 & 26,0 & 0,7759 & 0,1209 & 0,8969 & 3,7311 & 618,45 & 96,38 & 714,83 & 2973,69 \\
\hline 42 & 833 & 13 & 10 & 1,1218 & 0,1838 & 1,3056 & 5,4315 & 934,48 & 153,13 & $1.087,61$ & 4524,46 \\
\hline 43 & 780 & 17 & 12,85 & 4,4551 & 0,5346 & 4,9898 & 20,7577 & $3.475,04$ & 417,03 & $3.892,07$ & 16191,01 \\
\hline 44 & 600 & 8 & 21,12 & 4,9905 & 0,7136 & 5,7042 & 23,7294 & $2.994,35$ & 428,17 & $3.422,52$ & 14237,68 \\
\hline
\end{tabular}




\begin{tabular}{|c|c|c|c|c|c|c|c|c|c|c|c|}
\hline 45 & 534 & 15 & 14,13 & 3,759 & 0,4573 & 4,2170 & 17,5428 & $2.041,52$ & 248,33 & $2.289,85$ & 9525,78 \\
\hline 46 & 638 & 6 & 15,33 & 2,294 & 0,2689 & 2,5632 & 10,6632 & $1.463,80$ & 171,57 & $1.635,37$ & 6803,14 \\
\hline 47 & 628 & 3 & 26,0 & 2,2515 & 0,3466 & 2,5982 & 10,8086 & $1.414,00$ & 217,7 & $1.631,70$ & 6787,87 \\
\hline 48 & 818 & 1 & 5,5 & 0,0086 & & 0,0086 & 0,0359 & 7,07 & & 7,07 & 29,41 \\
\hline 49 & 910 & 4 & 10,25 & 0,4766 & 0,0727 & 0,5494 & 2,2857 & 433,8 & 66,21 & 500 & 2080,00 \\
\hline 50 & 800 & 7 & 9,0 & 0,7193 & 0,0410 & 0,7604 & 3,1634 & 575,51 & 32,85 & 608,36 & 2530,78 \\
\hline 52 & 670 & 1 & 10,0 & 0,0117 & & 0,0117 & 0,0490 & 7,89 & & 7,89 & 32,82 \\
\hline 53 & 798 & 5 & 16,3 & 1,7913 & 0,2264 & 2,0177 & 8,3937 & $1.429,46$ & 180,69 & $1.610,15$ & 6698,22 \\
\hline 54 & 860 & 1 & 22,5 & 0,5725 & 0,1005 & 0,6730 & 2,7999 & 492,4 & 86,44 & 578,84 & 2407,97 \\
\hline 55 & 598 & 1 & 31,0 & 1,217 & 0,1955 & 1,4128 & 5,8776 & 727,99 & 116,92 & 844,91 & 3514,83 \\
\hline 56 & 530 & 1 & 95,0 & 2,7979 & 2,9090 & 5,7070 & 23,7412 & $2.076,11$ & $1.541,79$ & $3.024,73$ & 12582,88 \\
\hline 57 & 790 & 1 & 14,5 & 0,1639 & 0,0313 & 0,1953 & 0,8127 & 129,55 & 24,78 & 154,34 & 642,05 \\
\hline 58 & 608 & 1 & 15,5 & 0,0365 & 0,0202 & 0,0567 & 0,2360 & 22,2 & 12,29 & 34,49 & 143,48 \\
\hline 59 & 558 & 1 & 18,5 & 0,3835 & 0,0405 & 0,4241 & 1,7645 & 214,04 & 22,64 & 236,68 & 984,59 \\
\hline 61 & 808 & 9 & 13,72 & 1,3466 & 0,2597 & 1,6064 & 6,6828 & $1.088,12$ & 209,89 & $1.298,01$ & 5399,72 \\
\hline 62 & 812 & 7 & 7,93 & 0,1611 & & 0,1611 & 0,6702 & 130,83 & & 130,83 & 544,25 \\
\hline 63 & 880 & 1 & 13,0 & 0,1837 & 0,0085 & 0,1923 & 0,8000 & 161,73 & 7,52 & 169,25 & 704,08 \\
\hline 64 & 612 & 1 & 13,5 & 0,1696 & 0,0112 & 0,1808 & 0,7524 & 103,82 & 6,88 & 110,7 & 460,51 \\
\hline 65 & 804 & 3 & 10,5 & 0,0816 & 0,014 & 0,0962 & 0,4002 & 65,67 & 11,68 & 77,35 & 321,78 \\
\hline 67 & 611 & 2 & 7,0 & 0,0289 & 0,0071 & 0,0360 & 0,1499 & 17,66 & 4,36 & 22,02 & 91,60 \\
\hline 68 & 700 & 2 & 20,5 & 1,1356 & 0,1776 & 1,3133 & 5,4636 & 794.98 & 124,39 & 919,37 & 3824,58 \\
\hline 69 & 1018 & 3 & 22,5 & 2,7602 & 0,4794 & 3,2397 & 13,4772 & $2.809,97$ & 488,08 & $3.298,05$ & 13719,89 \\
\hline 70 & 1003 & 8 & 31,19 & 9,6149 & 1,6544 & 11,2694 & 46,8807 & $9.643,79$ & $1.659,44$ & $11.303,23$ & 47021,44 \\
\hline 71 & 1053 & 4 & 33,87 & 4,4073 & 1,5374 & 5,9447 & 24,7301 & $4.640,94$ & $1.618,89$ & $6.259,83$ & 26040,89 \\
\hline 73 & 374 & 12 & 20,04 & 5,9201 & 1,0835 & 7,0037 & 29,1354 & $2.214,15$ & 405,24 & $2.619,39$ & 10896,66 \\
\hline 74 & 320 & 1 & 30,0 & 1,0602 & 0,1106 & 1,1709 & 4,8712 & 339,29 & 35,42 & 374,71 & 1558,79 \\
\hline 75 & 403 & 1 & 19,0 & 0,3805 & 0,0094 & 0,3899 & 1,6223 & 153,35 & 3,81 & 157,16 & 653,79 \\
\hline 76 & 512 & 2 & 11,5 & 0,1061 & & 0,1061 & 0,4417 & 54,37 & & 54,37 & 226,18 \\
\hline 77 & 588 & 1 & 14,0 & 0,2634 & 0,0184 & 0,2823 & 1,1747 & 155,17 & 10,88 & 166,05 & 690,77 \\
\hline Total & & 341 & & 105,09 & 19,50 & 124,60 & 518,33 & 80.807 & 14.786 & 95.594 & 397.674 \\
\hline
\end{tabular}

Em relação à biomassa lenhosa, foi registrado um total de $95.594,76 \mathrm{~kg}$, sendo $84,5 \%$ da biomassa dos fustes e 15,5\% da biomassa dos galhos, que representam $397.674,20 \mathrm{~kg} \cdot \mathrm{ha}^{-1}$. Rolim et al. (2005) registraram, para um trecho da Mata Attântica, entre 241.000 e 437.000 kg.ha ${ }^{-1}$. Nesse sentido, o resultado encontrado se enquadra na informação registrada por Rolim et al. (2005).

As espécies que apresentaram maiores valores em biomassa foram Manilkara longifolia, com $11.303,23 \mathrm{~kg}$, Aspidosperma australe, com 7.690,01 kg, Manilkara rufula, com 6.259,83 kg, Maprounea guianensis, com 5.630,33 kg, e Tapira guianensis, com 4.957,95 kg, perfazendo, as cinco espécies, $35.841,35 \mathrm{~kg}$.

Observa-se que as cinco espécies com maior volume de madeira acumularam 42,08 $\mathrm{m}^{3}$, que corresponderam a $33,77 \%$ do total do volume obtido. As cinco espécies com as maiores quantidades de biomassa $(35.841,35 \mathrm{~kg})$ perfizeram $9 \%$ do total da biomassa calculada.

Para a área da mata secundária em regeneração natural, foi encontrado um volume de madeira total de $22,49 \mathrm{~m}^{3}$ (Tabela 3), correspondendo para os fustes e galhos $87,55 \%$ e $12,45 \%$, respectivamente. O volume total por hectare foi de $93,59 \mathrm{~m}^{3}$, inferior ao valor encontrado por Drumond e Meira Neto (1999), que registraram, em uma área da Mata Atlântica, após 25 anos de regeneração natural, um volume de madeira de $205,3 \mathrm{~m}^{3} \cdot \mathrm{ha}^{-1}$.

Cinco espécies da área da mata secundária apresentaram um volume de madeira superior a $1 \mathrm{~m}^{3}$, Cecropia catarinensis $9,65 \mathrm{~m}^{3}$ (43\% do total volumétrico), Miconia cinnamomifolia $5,53 \mathrm{~m}^{3}$ (24,5\%), Piptocarpha macropoda 2,36 $\mathrm{m}^{3}(10,5 \%)$, Miconia multiflora $2,18 \mathrm{~m}^{3}(9,7 \%)$ e Vochysia bifalcata $1,41 \mathrm{~m}^{3}(6,3 \%)$. Essas cinco espécies, com 139 indivíduos arbóreos (71\% do total das árvores), acumularam $94 \%$ do total do volume de madeira.

$\mathrm{Na}$ área da mata secundária com 33 anos de idade, foram registrados $11.333,53 \mathrm{~kg}$ de biomassa dos fustes das 14 espécies. Nos galhos correspondentes das 195 árvores, foram contabilizados $1.768,89 \mathrm{~kg}$, perfazendo assim um total de $13.102,42 \mathrm{~kg}$ nos $2.400 \mathrm{~m}^{2}$ estudados, que correspondem a $54.631,22 \mathrm{~kg} \mathrm{ha}^{-1}$. Segundo o Serviço Florestal Brasileiro (SFB, 2010), o estoque de biomassa aérea no Bioma Mata Atlântica é em média de 66 toneladas por hectare. 
Tabela 3. Parâmetros dasométricos das espécies arbóreas encontradas na formação da mata secundária na Reserva Biológica Sítio Palmares (ES). Numeração da espécie extraída da tabela 1.

Table 3. Dendrometrical parameters of the woody species meet on the secondary forest formation at Reserva Biológica Sitio Palmares. Species number took from table 1.

\begin{tabular}{|c|c|c|c|c|c|c|c|c|c|c|c|}
\hline \multirow[b]{2}{*}{ Espécie } & \multicolumn{2}{|l|}{ Densidade } & \multirow{2}{*}{$\begin{array}{c}\text { DAP } \\
\text { médio } \\
(\mathrm{cm})\end{array}$} & \multicolumn{4}{|c|}{ Volume } & \multicolumn{4}{|c|}{ Biomassa } \\
\hline & $\begin{array}{c}\text { básica } \\
\left(\mathbf{k g . m ^ { 3 }}\right)\end{array}$ & $\mathbf{n}$ & & $\begin{array}{c}\text { Fuste } \\
\left(\mathbf{m}^{3}\right)\end{array}$ & $\begin{array}{c}\text { Galhos } \\
\left(\mathbf{m}^{3}\right)\end{array}$ & $\begin{array}{c}\text { Total } \\
\left(\mathbf{m}^{3}\right)\end{array}$ & $\begin{array}{c}\text { Madeira } \\
\left(\mathrm{m}^{3} \mathbf{h a}^{-1}\right)\end{array}$ & $\begin{array}{c}\text { Fuste } \\
\text { (kg) }\end{array}$ & $\begin{array}{c}\text { Galhos } \\
(\mathbf{k g})\end{array}$ & $\begin{array}{c}\text { Total } \\
\text { (kg) }\end{array}$ & $\begin{array}{c}\text { Seca } \\
\left({\left.\mathrm{kg} . h a^{-1}\right)}^{-}\right.\end{array}$ \\
\hline 02 & 800 & 3 & 10,83 & 0,1418 & 0,0033 & 0,1451 & 0,6039 & 113,47 & 2,67 & 116,14 & 483,16 \\
\hline 08 & 798 & 1 & 12,00 & 0,0376 & & 0,0376 & 01568 & 30,08 & & 30,08 & 125,15 \\
\hline 10 & 480 & 18 & 11,97 & 2,1826 & 0,1846 & 2,3672 & 9,8479 & $1.047,68$ & 88,62 & $1.136,30$ & $4.727,02$ \\
\hline 11 & 701 & 3 & 11,83 & 0,0812 & 0,0352 & 0,1165 & 0,4849 & 56,98 & 24,74 & 81,72 & 339,95 \\
\hline 26 & 720 & 8 & 10,31 & 0,2502 & 0,0278 & 0,2781 & 1,1569 & 180,19 & 20,05 & 200,24 & 833,00 \\
\hline 29 & 920 & 2 & 5,00 & 0,0157 & & 0,0157 & 0,0653 & 14,45 & & 14,45 & 60,12 \\
\hline 35 & 807 & 23 & 7,06 & 0,2651 & 0,0015 & 0,2667 & 1,1094 & 213,95 & 1,28 & 215,23 & 895,35 \\
\hline 43 & 780 & 2 & 8,25 & 0,0173 & 0,0035 & 0,0208 & 0,0868 & 13,51 & 2,77 & 16,28 & 67,73 \\
\hline 49 & 910 & 58 & 13,31 & 4,5505 & 0,9873 & 5,5378 & 23,0373 & $4.140,98$ & 898,44 & $5.039,42$ & $20.964,01$ \\
\hline 50 & 800 & 1 & 14,00 & 0,2638 & 0,0408 & 0,3047 & 1,2676 & 211,12 & 32,66 & 243,78 & $1.014,12$ \\
\hline 51 & 549 & 25 & 12,20 & 1,7251 & 0,4627 & 2,1879 & 9,1018 & 947,12 & 254,06 & $1.201,18$ & $4.996,91$ \\
\hline 53 & 798 & 5 & 8,50 & 0,0823 & 0,0035 & 0,0859 & 0,3575 & 65,75 & 2,83 & 68,58 & 285,30 \\
\hline 60 & 769 & 1 & 11,00 & 0,0483 & 0,0023 & 0,0507 & 0,2111 & 37,20 & 1,83 & 39,03 & 162,37 \\
\hline 66 & 760 & 1 & 5,50 & 0,0034 & & 0,0034 & 0,0143 & 2,63 & & 2,63 & 10,93 \\
\hline 72 & 440 & 1 & 5,00 & 0,0031 & & 0,0031 & 0,0130 & 1,38 & & 1,38 & 5,75 \\
\hline 75 & 403 & 40 & 15,49 & 8,8402 & 0,8166 & 9,6568 & 40,1726 & $3.562,63$ & 329,09 & $3.891,72$ & $16.189,57$ \\
\hline 77 & 588 & 3 & 24,33 & 1,1809 & 0,2379 & 1,4189 & 5,9027 & 694,41 & 139,92 & 834,33 & $3.470,80$ \\
\hline Total & & 195 & & 19,69 & 2,80 & 22,49 & 93,59 & 11.333 & 1.768 & 13.102 & 54.631 \\
\hline
\end{tabular}

\section{CONCLUSÕES}

- A área da mata secundária, após 33 anos, mostra estar num estado inicial de secundarização, pois ainda não atingiu a fase próxima ao clímax, quando comparados os resultados com a mata primária estudada, notadamente no que tange ao número de espécies e indivíduos.

- Os resultados obtidos da estrutura e o estado de desenvolvimento da mata primária corroboram as informações da literatura, deduzindo-se que a formação vegetal estudada, mesmo com a existência de possíveis ações antrópicas, encontra-se em satisfatório estado de conservação.

\section{REFERENCIAS}

ALVES JÚNIOR, F. T.; SILVA BRANDÃO, C. F. L.; ROCHA, K. D. da; SILVA, J. T. da; MARANGON, L. C.; FERREIRA, R. L. C. Estrutura diamétrica e hipsométrica do componente arbóreo de um fragmento de Mata Atlântica, Recife - PE. Cerne, Lavras, v. 13, n. 1, p. 83 - 95, 2007.

APARÍCIO, W. C. da S.; MARANGON, L. C.; FERREIRA, R. L. C.; FELICIANO, A. L. P.; APARÍCIO, P. S.; COSTA JÚNIOR, R. F. Estrutura da regeneração natural de espécies arbóreas em um fragmento de Mata Atlântica, Pernambuco. Revista Brasileira de Ciências Agrárias, Recife, v. 6, n. 3, p. 483 - 488. 2011.

BEEK, R. A. D.; SÁENZ, G. Manejo forestal baseado en la regeneración natural del bosque: estúdio de caso en los robledales de altura de la Cordillera Talamanca, Costa Rica. Turrialba: CATIE, 1992. 48 p. (Informe Técnico, 200).

BORÉM, R. A. T.; OLIVEIRA-FILHO, A. T. Fitossociologia do estrato arbóreo em uma topossequência alterada de Mata Atlântica no município de Silva Jardim - RJ, Brasil. Árvore, Viçosa, v. 26, n. 6, p. 727 742, 2002.

BRAGA, C. S.; BARBOSA, V. H. B.; GAZZOLA, C. E.; DIAS, H. C. T. Relação entre área basal e a precipitação efetiva em um fragmento de Mata Atlântica na zona da mata norte mineira. In: SEMINÁRIO DE RECURSOS HÍDRICOS DA BACIA DO PARAÍBA DO SUL: RECUPERAÇÃO DE ÁREAS DEGRADADAS, SERVIÇOS AMBIENTAIS E SUSTENTABILIDADE, II. Anais do... Taubaté, 2009. p. $661-665$. 
BURGER, D. M.; DELITTI, W. B. C. Allometric models for estimating the phytomass of a secondary Atlantic Forest área of southeastern Brazil. Biota Neotropica, Campinas, v. 8, v. 4, p. 131 - 136. 2008.

CENTRO DE INFORMACIÓN DE EXPERIENCIAS DE ADAPTACIÓN Y CRECIMIENTO DE ESPECIES FORESTALES EN CHILE (CIAF). Glosario de términos. Disponível em: <http://www.infor.cl/ciacef/glosario.htm\#L>. Acesso em 01/03/2012.

CHICHORRO, J. F.; RESENDE, J. L. P.; LEITE, H. G. Equações de volume e de taper para quantificar multiprodutos da madeira em floresta atlântica. Árvore, Viçosa, v. 27, n. 6, p. 799 - 809. 2003.

CUNHA, G. de M.; GAMA-RODRIGUES, A. C.; GAMA-RODRIGUES, E. F.; VELlOSO, A. C. X. Biomassa e estoque de carbono e nutrientes em florestas montanas da Mata Atlântica na região norte do estado de Rio de Janeiro. Rev. Bras. Ciência do Solo, Viçosa, v. 33, p. 1175 - 1185. 2009.

CRUZ JOHNSON, P.; HONEYMAN LUCCHINI, P.; CABALLERO TAPIA, C. Propuesta metodológica de ordenación forestal, aplicación a bosques de lenga en la XI región. Bosque, Valdívia, v. 26, n. 2, p. 57 - 70. 2005.

DELDUQUE, M.; MILANEZ, F.; RIBEIRO, H.; NOGUEIRA-NETO, P. Mata Atlântica. Porto Alegre: Terrabrasil, 2008. 260 p.

DRUMOND, M. A.; MEIRA NETO, J. A. Composições florística e fitossociológica de uma mata secundária de um trecho da Mata Atlântica. Ciência Rural, Santa Maria, v. 29, n. 4, p. 657 - 661, 1999.

HAWLEY, R.; SMITH, D. F. Silvicultura práctica. España: Ediciones Omega, 1982. 544 p.

IMAÑA-ENCINAS, J.; SILVA, G. F. da; KISHI, I. T. Variáveis dendrométricas. Brasília: Universidade de Brasília, Depto. Engenharia Florestal, 2002. 102 p. (Comunicações Técnicas Florestais, v. 4, n. 1).

INSTITUTO CAPIXABA DE PESQUISA, ASSISTÊNCIA TÉCNICA E EXTENSÃO RURAL (INCAFER). Disponível em: <http://hidrometeorologia.incaper.es.gov.br/?pagina=regiao_serran>. Acesso em 01/10/2011.

KENT, M.; COKER, P. Vegetation description and analysis. Chichester: Willey, 1994. 363 p.

KURTZ, B. C.; ARAÚJO, D. S. D. de. Composição florística e estrutura do componente arbóreo de um trecho de Mata Atlântica na Estação Ecológica Estadual do Paraíso, Cachoeiras de Macacu, Rio de Janeiro, Brasil. Rodriguésia, Rio de Janeiro, v. 51, n. 78/79, p. 69 - 112. 2000.

MAGURRAN, A. E. Ecological diversity and its measurement. London: Chapman and Hall, 1988. 256 p.

MARANGON, L. C.; SOARES, J. J.; FELICIANO, A. L.; SILVA BRANDÃO, C. F. L. Regeneração natural em um fragmento de floresta estacional semidecidual em Viçosa, Minas Gerais. Árvore, Viçosa, v. 32, n. 1, p. 183 - 191, 2008.

MUELLER-DOMBOIS, D.; ELLENBERG, H. Aims and methods of vegetation ecology. New York: Willey, 1974. 547 p.

OLIVEIRA, R. R. Ação antrópica e resultantes sobre a estrutura e composição da Mata Atlântica na Ilha Grande, RJ. Rodriguésia, Rio de Janeiro, v. 53, n. 82, p. 33 - 58. 2002.

PANOSO, L. A. (coord.). Levantamento de reconhecimento dos solos do Estado do Espírito Santo. Rio de Janeiro: EMBRAPA, 1978. 461 p. (Boletim Técnico do Serviço Nacional de Levantamento e Conservação de Solos, 45).

ROLIM, S. G.; COUTO, H. T. Z. do; JESUS, R. M. de. Modelos volumétricos para a Floresta Nacional do Tapirapé-Aquiri, Serra dos Carajás (PA). Acta Amazonica, Manaus, v. 36, n. 1, p. 107 - 114. $2006 \mathrm{a}$.

ROLIM, S. G.; IVANAUSKAS, N. M.; RODRIGUES, R. R.; NASCIMENTO, M. T.; GOMES, J. M. L.; FOLLI, D. A.; COUTO, H. Z. do. Composição florística do estrato arbóreo da floresta estacional semidecidual na planície aluvial do rio Doce, Linhares, ES, Brasil. Acta Botanica Brasilica, Feira de Santana, v. 20, n. 3, p. 549 - 561, 2006b.

ROLIM, S. G.; JESUS, R. M.; NASCIMENTO, H. E. M.; COUTO, H. T. Z; CHAMBERS, J. Q. Biomass change in an Atlantic tropical moist forest: the ENSO effect in permanent sample plots over 22-year period. Oecologia, Berlin, v. 142, n. 2, p. 238 - 246, 2005. 
SERVIÇO FLORESTAL BRASILEIRO (SFB). Florestas do Brasil em resumo. 2010: dados de 20052010. Brasília: SFB, 2010. 152 p.

SILVA, M. A. M.; GUERRA, T. N. F.; MENDES, P. G. de A.; BARBOSA, M. D.; ROCHA, K. D.; CHAVES, L. de F. de C. Diversidade da regeneração natural de diferentes amostragens em um fragmento de floresta atlântica, Recife, PE. In: JORNADA DE ENSINO, PESQUISA E EXTENSÃO, IX. 2009, Recife, UFRPE. 3 p. Resumos...

SILVEIRA, P.; KOEHLER, H. S.; SANQUETTA, C. R.; ARCE, J. E. O estado da arte na estimativa de biomassa e carbono em formações florestais. Floresta, Curitiba, v. 38, n. 1, p. 185 - 206. 2008.

SOS MATA ATLÂNTICA. Atlas dos remanescentes florestais da Mata Atlântica: período de 2000 a 2005. São Paulo: Editora Fundação SOS Mata Atlântica, 2008. 157 p.

SOUZA, A. L.; JESUS, R. M. Equações de volume e fator de forma para espécies da Mata Atlântica ocorrentes na reserva florestal da Companhia Vale do Rio Doce, Linhares - ES. Árvore, Viçosa, v. 15, n. 3, p. 257 - 273. 1991.

TROPICOS. Disponível em: <http://tropicos.org>. Acesso em 24/01/2010.

VIEIRA, S. A.; ALVES, L. F.; AIDAR, M.; ARAÚJO, L. S.; BAKER, T.; BATISTA, J. L. F.; CAMPOS, M. C. R.; CAMARGO, P. B.; CHAVE, J.; DELITTI, W. B.; HIGUCHI, N.; HONÓRIO, E.; JOLY, C. A.; KELLER, M.; MARTINELLI, L. A.; DE MATTOS, E. A.; METZKER, T.; PHILLIPS, O.; SANTOS, F. A. M.; SHIMABUKURO, M. T.; SILVEIRA, M.; TRUMBORE, S. E. Estimation of biomass and carbon stocks: the case of the Atlantic Forest. Biota Neotropica, Campinas, v. 8, n. 2, p. 21 - 29. 2008. 